\title{
Abnormalities in the basement membrane structure promote basal keratinocytes in the epidermis of hypertrophic scars to adopt a proliferative phenotype
}

\author{
SHAOWEI YANG ${ }^{1,2^{*}}$, YEXIAO SUN ${ }^{2,3 *}$, ZHIJUN GENG $^{2}$, KUI MA $^{2}$, XIAOYAN SUN $^{1}$ and XIAOBING FU ${ }^{1,2}$ \\ ${ }^{1}$ Wound Healing and Cell Biology Laboratory, Institute of Basic Medical Science, Trauma Center \\ of Postgraduate Medical School, Chinese PLA General Hospital, Beijing 100853; ${ }^{2}$ Key Research Laboratory \\ of Tissue Repair and Regeneration of PLA, and Beijing Key Research Laboratory of Skin Injury, \\ Repair and Regeneration, The First Affiliated Hospital to the Chinese PLA General Hospital, Beijing 100048; \\ ${ }^{3}$ Graduate School of Inner Mongolia Medical University, Hohhot 010110, P.R. China
}

Received December 9, 2015; Accepted February 29, 2016

DOI: $10.3892 /$ ijmm.2016.2519

\begin{abstract}
The majority of studies on scar formation have mainly focused on the dermis and little is known of the involvement of the epidermis. Previous research has demonstrated that the scar tissue-derived keratinocytes are different from normal cells at both the genetic and cell biological levels; however, the mechanisms responsible for the fundamental abnormalities in keratinocytes during scar development remain elusive. For this purpose, in this study, we used normal, wound edge and hypertrophic scar tissue to examine the morphological changes which occur during epidermal regeneration as part of the wound healing process and found that the histological structure of hypertrophic scar tissues differed from that of normal skin, with a significant increase in epidermal thickness. Notably, staining of the basement membrane (BM) appeared to be absent in the scar tissues. Moreover, immunofluorescence staining for cytokeratin (CK)10, CK14, CK5, CK19 and integrin- $\beta 1$ indicated the differential expression of cell markers in the epidermal keratinocytes among the normal, wound edge and hypertrophic scar tissues, which corresponded with the
\end{abstract}

Correspondence to: Dr Xiaoyan Sun, Wound Healing and Cell Biology Laboratory, Institute of Basic Medical Science, Trauma Center of Postgraduate Medical School, Chinese PLA General Hospital, 28 Fu Xing Road, Beijing 100853, P.R. China

E-mail: yanzisun1979@sina.com

Dr Xiaobing Fu, Key Research Laboratory of Tissue Repair and Regeneration of PLA, and Beijing Key Research Laboratory of Skin Injury, Repair and Regeneration, The First Affiliated Hospital to the Chinese PLA General Hospital, 51 Fu Cheng Road, Beijing 100048, P.R. China

E-mail: fuxiaobing@vip.sina.com

*Contributed equally

Key words: basement membrane, keratinocyte, proliferation, wound healing, scar altered BM structures. By using a panel of proteins associated with BM components, we validated our hypothesis that the BM plays a significant role in regulating the cell fate decision of epidermal keratinocytes during skin wound healing. Alterations in the structure of the BM promote basal keratinocytes to adopt a proliferative phenotype both in vivo and in vitro.

\section{Introduction}

The skin serves as the physical and chemical barrier between the interior body and the exterior environment, which consists of an underlying dermis of mesodermal origin and an overlaying epidermis of ectodermal origin. Although these two layers perform different functions, they communicate in various ways and at different levels. The vital barrier function of skin is primarily provided by its upper stratified epidermis, composed of proliferating basal and differentiated suprabasal keratinocytes (1). To complete these functions, stem cells in the basal layer are capable of self-renewal throughout life and they produce daughter cells [transit-amplifying cells (TAs)] that undergo differentiation (2). Thus, the balance between the proliferation and differentiation of the basal keratinocytes is essential for epidermal integrity, and it ensures the occurrence of tissue renewal which is necessary to complete normal physiological functions. In addition to maintaining tissue homeostasis, stem cells residing in the epidermis and hair follicles also participate in the repair of the epidermis following injury $(3,4)$. In the skin, different phases of wound healing evolve in dynamic interactions between epidermal cells, dermal cells and bone marrow-derived cells. Keratinocytes represent the 'first line of defense' of the body against the outside environment. Therefore, they are the first responders to injury. With the release of inflammatory cytokines and the contraction of collagenous fibers, the basal epidermal keratinocytes are activated and migrate into the wound area, where they proliferate and differentiate until the cellular content of the tissue has been renewed (5). A number of observations have suggested that the epidermis has an effect on the pathology of cutaneous scars $(6,7)$. In burn patients, hypertrophic scars occur more frequently when wounds heal by secondary 
intention, whereas early skin grafting appears to suppress scar formation (8). Funayama et al demonstrated that keloid-derived fibroblasts co-cultured with keloid-derived keratinocytes were significantly more proliferative and resistant to apoptosis than those co-cultured with normal skin-derived keratinocytes (9). In a similar co-culture system, it was found that keloid-derived keratinocytes promoted increased collagen production, as well as the increased proliferation of normal fibroblasts (10). In particular, keloid-derived keratinocytes have been shown to exhibit the increased expression of a set of histone genes essential for DNA replication in comparison with the expression levels in normal cells, and keratinocytes in the epidermis of hypertrophic scars have entered an alternative differentiation pathway and express a proliferative phenotype $(11,12)$. These results suggest that the balance between the proliferation and differentiation of keratinocytes is dysregulated in scar tissues, and that fundamental abnormalities in keratinocytes may play a more important role in skin regeneration and its pathogenesis than was previously appreciated.

In the present study, normal, wound edge and hypertrophic scar tissues were obtained and analysed using histological and immunofluorescence methods to investigate the differences in morphology, as well as keratin expression profiles during epidermal regeneration. Our results indicated that there was a variation in the keratin expression profiles in the epidermal keratinocytes from normal, wound edge and hypertrophic scar tissues, which corresponded with abnormalities in the structure of the basement membrane (BM). By using a panel of antibodies to BM components, we validated our hypothesis and determined that the $\mathrm{BM}$ was a positive regulator of the recruitment of precursor-like cells to the basal layer of the epidermis. Our data indicate that alterations in the structure of the BM promotes basal keratinocytes to adopt a proliferative phenotype both in vivo and in vitro.

\section{Materials and methods}

Tissues samples. Hypertrophic scar tissue (4 females and 3 males; age range 18-40 years) and wound edge tissue ( 3 females and 5 males; age range, 18-40 years) samples were obtained from patients who had undergone prior reconstructive burn surgery. Normal skin tissue (4 females and 3 males; age range, 18-40 years) adjacent to the scar excision during surgery was used as a control. Foreskins were also obtained from males undergoing circumcision (10 males; age range, 18-20 years). The present study was approved by the Ethics Committee of the Chinese PLA General Hospital (Beijing, China), and written informed consent was obtained from all individuals prior to obtaining the samples.

Immunofluorescence staining. Normal skin, wound edge and hypertrophic scar tissue samples were fixed in $10 \%$ buffered formalin, dehydrated through ethanol solutions with increased concentration successively, and finally embedded in paraffin, respectively. Paraffin-embedded tissues were cut into 4- $\mu \mathrm{m}$-thick sections for staining with hematoxylin and eosin (H\&E), Masson's trichrome, and methenamine silver (all from Zhongshan Golden Bridge, Beijing, China). Images were captured using an optical microscope (BX53; Olympus, Tokyo, Japan). To perform immunofluorescence staining, the sections were fixed in $4 \%$ paraformaldehyde for $30 \mathrm{~min}$, and then permeabilized in $0.2 \%$ Triton X-100 (T8787; Sigma-Aldrich, St. Louis, MO, USA) in phosphate-buffered saline (PBS) for $10 \mathrm{~min}$. The sections were incubated with primary antibodies at $4^{\circ} \mathrm{C}$ overnight and then with secondary antibodies for $2 \mathrm{~h}$ at room temperature. The following primary antibodies were used: mouse anti-human cytokeratin (CK)10 (1:200, ab111447) and rabbit anti-human CK14 (1:200, ab7800) (both from Abcam, Cambridge, MA, USA), rabbit anti-human CK 5 (1:200, ZA-0518; Zhongshan Goldenbridge, Beijing, China), mouse anti-human CK19 (1:200, ab53119; Abcam), rabbit antihuman integrin- $\beta 1$ (1:200, PB0063; Boster, Wuhan, China); mouse anti-human integrin- $\beta 4$ (1:200, ab128068; Abcam), mouse anti-human laminin (1:200, ZM-0181; Zhongshan Goldenbridge), rabbit anti-human laminin-5 (1:200, ab14509; Abcam) and mouse anti-human collagen IV (1:200, ZM-0081; Zhongshan Goldenbridge). The following secondary antibodies were used: Alexa-Fluor 488-conjugated anti-mouse (1:200, ab150117) and Alexa-Fluor 594-conjugated anti-rabbit (1:200, ab150080) (both from Abcam). The nuclei were stained with DAPI (H-1200; Vector Laboratories, Burlingame, CA, USA). Immunofluorescence images were captured using a confocal laser scanning microscope (SP8; Leica, Solms, Germany).

Cell culture and treatment. Primary human epidermal keratinocytes (HEKs) were isolated from the male foreskins as previously described (13), with minor modifications. The human immortalized keratinocyte (HaCaT) cells were purchased from the China Infrastruture of Cell Line Resources (3111C0001CCC000373; Beijing, China). Both the HEKs and the HaCaT cells were incubated in EpiLife medium (M-EPI-500-CA; Invitrogen Life Technologies, Carlsbad, CA, USA) supplemented with $0.06 \mathrm{mM} \mathrm{Ca}^{2+}, 1 \%$ EpiLife defined growth supplement (S-001-5; Invitrogen Life Technologies), and $1 \%$ penicillin/streptomycin (P1400; Solarbio, Beijing, China). To investigate the roles of the BM in regulating keratinocyte behavior in vitro, the HEKs and HaCaT cells were plated in $60 \mathrm{~mm}$-dishes coated with MaxGel ECM (E0282) and collagen type IV (C7521) (both from Sigma-Aldrich), respectively. For $\mathrm{Ca}^{2+}$ treatment, the cells were incubated in EpiLife medium supplemented with $1.5 \mathrm{mM} \mathrm{Ca}^{2+}$ for 0,12 and $24 \mathrm{~h}$. The MaxGel ECM contains human extracellular matrix (ECM) components including collagens, laminin, fibronectin, tenascin and elastin, as well as a number of proteoglycans and glycosaminoglycans. The cells not treated with $\mathrm{Ca}^{2+}$ were used as controls.

Reverse transcription-quantitative (real-time) $P C R$ $(R T-q P C R)$. Total RNA was isolated from the cells after they were subjected to the various treatments using TRIzol reagent (15596-026; Invitrogen, Carlsbad, CA, USA) and then cDNA was synthesized using GoScript reverse transcriptase (A5001; Promega, Madison, WI, USA) according to the manufacturer's instructions. The primer sequences used for gene amplification are listed in Table I. qPCR reactions were performed with GoTaq qPCR Master Mix (A6001; Promega) using an ABI 7500 Real-Time PCR system and software (Applied Biosystems, Foster City, CA, USA). The reaction protocol consisted of the following cycles: $95^{\circ} \mathrm{C}$ for $15 \mathrm{sec}, 55^{\circ} \mathrm{C}$ for $30 \mathrm{sec}$, and $72^{\circ} \mathrm{C}$ for $30 \mathrm{sec}$ for 40 cycles of PCR amplification. 
Table I. Primers used for RT-qPCR in this study.

\begin{tabular}{lll}
\hline Transcript & & Primer sequences $\left(5^{\prime} \rightarrow 3^{\prime}\right)$ \\
\hline CK10 & Sense: & GGCAAAATCAAGGAGTGGTATG \\
& Antisense: & GAAGCAGGATGTTGGCATTATC \\
CK14 & Sense: & GAAGTGAAGATCCGTGACTGGT \\
& Antisense: & GTGGCTGTGAGAATCTTGTTCC \\
CK19 & Sense: & GCCACTACTACACGACCATCCA \\
& Antisense: & AGAGCCTGTTCCGTCTCAAACT \\
GAPDH & Sense: & TGAAGGTCGGAGTCAACGGAT \\
& Antisense: & CTGGAAGATGGTGATGGGATT
\end{tabular}

Western blot analysis. Total protein was isolated from the cells and $20 \mu \mathrm{g}$ of protein was dissolved in substrate-soluble buffer. The proteins were then separated by SDS-PAGE and transferred onto polyvinylidene difluoride (PVDF) membranes (IPFL00010; Millipore, Billerica, MA, USA). After blocking, the blots were probed with the primary antibodies overnight at $4^{\circ} \mathrm{C}$. The antibodies used for western blot analysis included CK10 (1:1000, ab111447), CK14 (1:500, ab7800), CK19 (1:500, ab53119), integrin- $\beta 4$ (1:500, ab128068), and mouse anti-human $\beta$-actin antibody (1:5000, ab6276) (all from Abcam). After washing, the membranes were incubated with secondary antibodies, which were goat anti-rabbit and goat anti-mouse IgG conjugated to horseradish peroxidase (sc-2004, 1:1000; sc-2005, 1:1000; Santa Cruz Biotechnology, Santa Cruz, CA, USA). Immunoreactive bands were detected by enhanced chemiluminescence (ECL) kit (PE-0010-100; Solarbio, Beijing, China) and imaged using the ImageQuant LAS 4000 system (GE Healthcare Bio-Sciences, Pittsburgh, USA).

Statistical analysis. All experiments were repeated at least 3 times, unless otherwise indicated. Data are presented as the means \pm standard deviation (SD). Statistical analysis was performed using SPSS 20.0 software. Data comparison was performed using the independent Student's t-test and one-way ANOVA followed by the LSD test. A P-value $<0.05$ was considered to indicate a statistically significant difference.

\section{Results}

Morphological differences in normal, wound edge and hypertrophic scar tissues. Skin wound repair is a precise remodeling process, which is divided classically into four overlapping phases and involves the interaction of many different tissues and cell lineages (5). In chronic wounds, however, the normal healing process is interrupted, which results in a pathological cycle of inflammation and protease release leading to the development of a pathological scar. In this study, to evaluate the morphological changes occurring in the regenerating epidermis during skin wound healing, specimens of normal, wound edge and hypertrophic scar tissues were obtained in order to perform routine H\&E and Masson's trichrome staining. In the normal skin samples, the histological structure of the skin was visible, showing layers of the epidermis and dermis. The epidermis contained 4 to 5 layers, in which keratinocytes differentiated and gradually matured into spinous cells, granular cells and cornified cells during their outward passage (Fig. 1A). Moreover, in the underlying dermis, fibroblasts made up loose connective tissue, which was rich in parallel collagenous fibers (Fig. 1D). In the tissues around the wound edge, however, the epidermis proliferated; it was comprised of multiple layers of keratinocytes of distinct morphology and was of visibly increased thickness (Fig. 1B and E). The epidermis and dermis interlocked through finger-like projections (called rete ridges), which increased the surface area of contact between the epidermis and the dermis. In both the normal and wound edge tissues, the basal keratinocytes were arranged as a regimented single layer of cuboidal or low columnar cells, which connected to the spinous cell layers above (Fig. 1A and B). The histological structure of the hypertrophic scar tissue differed from that of normal skin tissue by having a rich blood supply, and a thick epidermal layer (Fig. 1C and F). The dermis was composed largely of dense, disorganized connective tissue that includes tough collagenous fibers of an irregular shape and a larger diameter (Fig. 1F). To further clarify the differences in histological structure among the normal, wound edge and hypertrophic scar tissues, methenamine silver staining was performed to assess the formation of the BM, which indicated that the BM structure was detectable in the sections of the normal and wound edge tissues (Fig. 1G and H). In the scar tissues, however, BM staining was absent (Fig. 1I).

Differences in the expression of cell markers in epidermal keratinocytes in normal, wound edge and hypertrophic scar tissues. Given the morphological differences which were observed in the normal, wound edge and hypertrophic scar tissues, we examined whether the basal keratinocytes exhibited a different cellular behavior during scar formation. Initially, we performed immunofluorescence staining to detect the expression of CK10, CK14, CK5, CK19 and integrin- $\beta 1$ in the sections of normal, wound edge and scar tissues. Previous studies have verified that CK10 is a marker of differentiated keratinocytes, that CK14 and CK5 are markers of proliferating basal keratinocytes or TAs, and that CK19 is a putative marker of epidermal progenitor cells or stem cells in the skin (14-17). Integrin- $\beta 1$ is also thought to be a marker of precursor cells in the skin $(18,19)$. Our results indicated that CK10 was expressed in the outer layer of the epidermis in the normal and wound edge tissues (Fig. 2A and B), and in the suprabasal, terminally differentiating cells in the epidermis of the hypertrophic scar tissue (Fig. 2C). CK14 was expressed in both basal and suprabasal layers in the stratified epidermis of all three tissues (Fig. 2D-F), and there was an extensive distribution of CK14 in the multilayered epidermis of the scar tissue (Fig. 2F). The distribution pattern of CK5 was similar to that of CK14 (Fig. 2G-I), which indicated an increased number of proliferating cells in the hyperproliferative epidermis. Integrin- $\beta 1$ and CK19 were expressed in the basal layer of the normal epidermis (Fig. 2J and M) and in the basal and suprabasal layers of the wound edge epidermis (Fig. $2 \mathrm{~K}$ and $\mathrm{N}$ ), but were not detectable in the epidermis of the hypertrophic scar tissues (Fig. 2L and O). Collectively, these observations indicate that the keratin expression profile of basal keratinocytes differed between the normal, wound edge and hypertrophic scar tissues. Although fewer CK19-expressing cells were detected in the basal and suprabasal layers of the hypertrophic scar tissue (Fig. 2P), 


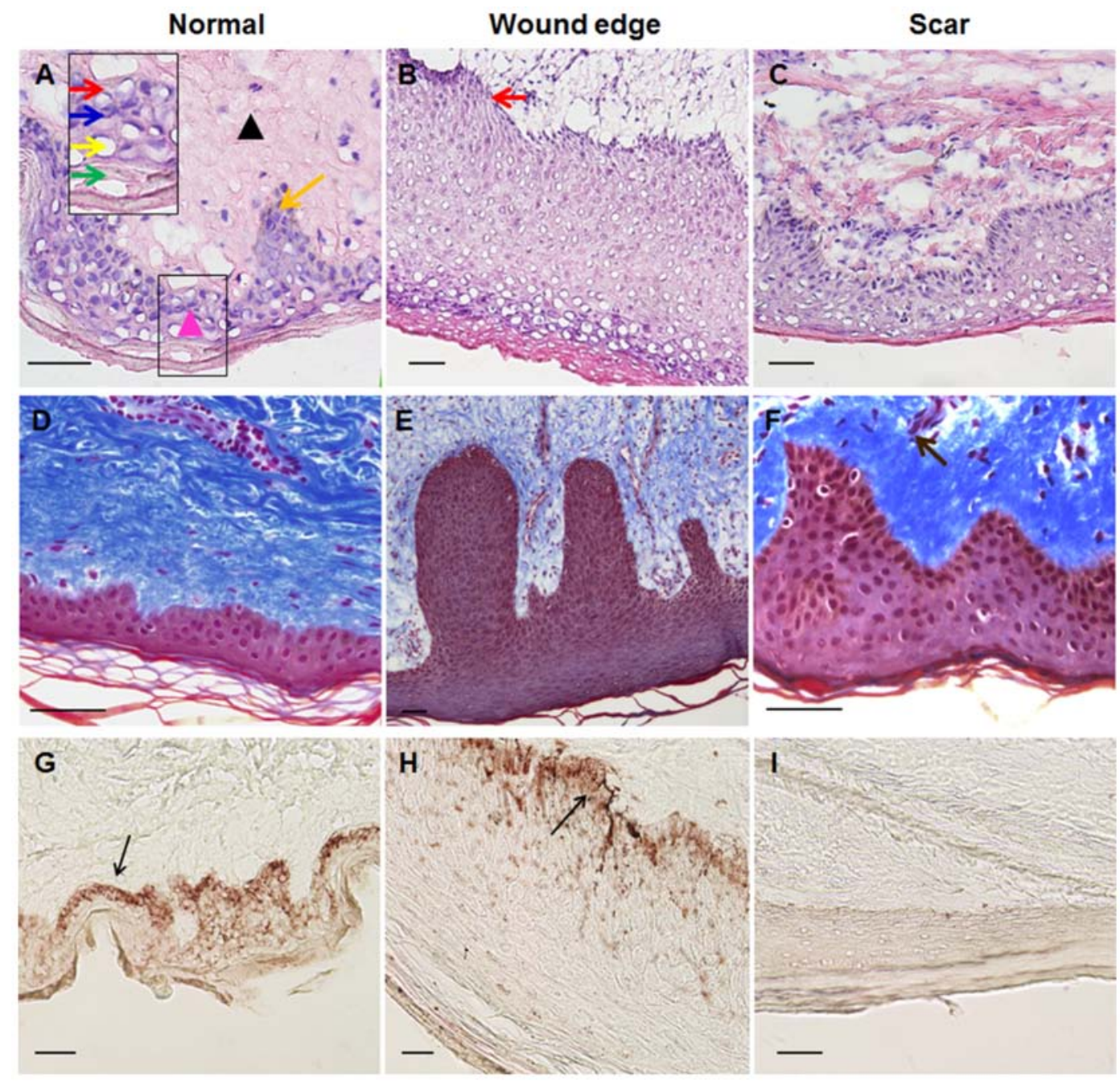

Figure 1. Morphological differences between normal, wound edge and hypertrophic scar tissues. (A-C) Hematoxylin and eosin (H\&E) staining was performed to visualize the histological structures in normal, wound edge and scar tissues. (A) In the normal skin sample, the histological structure of the skin was visible, showing layers of the epidermis and dermis. The epidermis contained 4 to 5 layers of keratinocytes. In the underlying dermis, fibroblasts made up loose connective tissue, which was rich in parallel collagenous fibers (D). Inset shows a close-up view of area within the black markings. Black triangle denotes dermis, while pink triangle denotes epidermis. Orange arrow denotes rete ridge. Red arrow denotes basal keratinocytes. Blue arrow denotes spinous cells. Yellow arrow denotes granular cells. Green arrows denote cornified cells. (B) In the wound edge tissue sample, the epidermis proliferated and was of increased thickness and was comprised of multiple layers of keratinocytes of distinct morphology. Red arrow denotes basal keratinocytes, which arranged as a regimented single layer of cuboidal or low columnar cells. (C) The histological structure of hypertrophic scar tissue differed from normal skin by having a rich blood supply, high mesenchymal density, and a thick epidermal layer. The dermis was composed largely of dense, disorganized connective tissue that includes tough collagenous fibers of an irregular shape and a larger diameter. (D-F) Masson's staining was performed to visualize the distribution of collagen fibers in (D) normal, (E) wound edge and (F) scar tissues. Brown arrow denotes tough collagens with rich blood supply. (G-I) Methenamine silver staining was performed to detect the basement membrane $(\mathrm{BM})$ structure in $(\mathrm{G})$ normal, $(\mathrm{H})$ wound edge and $(\mathrm{I})$ scar tissues. BM was present in normal and wound edge tissue, but absent in scar tissue. $(\mathrm{G}$ and $\mathrm{H})$ Black arrows denote the BM. All scale bars represent $50 \mu \mathrm{m}$.

a proliferative phenotype was revealed due to the increased expression of CK14 and CK5 in the multilayered epidermis.

Altered BM structure contributes to the differential keratin expression profile in epidermal keratinocytes in vivo. As a key component of the stem cell niche, the ECM not only anchors stem cells but also directs their fate (20). As mentioned above, the histological structure of the BM appeared to be absent in the hypertrophic scar tissue samples. Given the differences in the keratin expression profiles in the basal layer of normal, wound edge and hypertrophic scar epidermis, we then speculated that the structural abnormalities of the BM play a role in regulating the cell fate decision of basal keratinocytes during wound healing. To examine this hypothesis, immunofluorescence staining was performed to visualize the components of the BM in the normal, wound edge and hypertrophic scar tissue samples. Our results revealed that collagen IV expression was detected in the BM area in both normal (Fig. 3A) and wound edge (Fig. 3B) tissues. However, the formation of BM-like structures with an absence of collagen IV expression was observed in the epidermis of hypertrophic scar tissues (Fig. 3C). Although the expression of laminin was detected in all three tissue types (Fig. 3D-F), the double-labelling of laminin-5 and its receptor integrin- $\beta 4$ further verified our observation that the structure of the BM was altered in the epidermis of the hypertrophic scar tissues; negative staining of both laminin-5 and integrin- $\beta 4$ (Figs. 3I and 4G-I) 


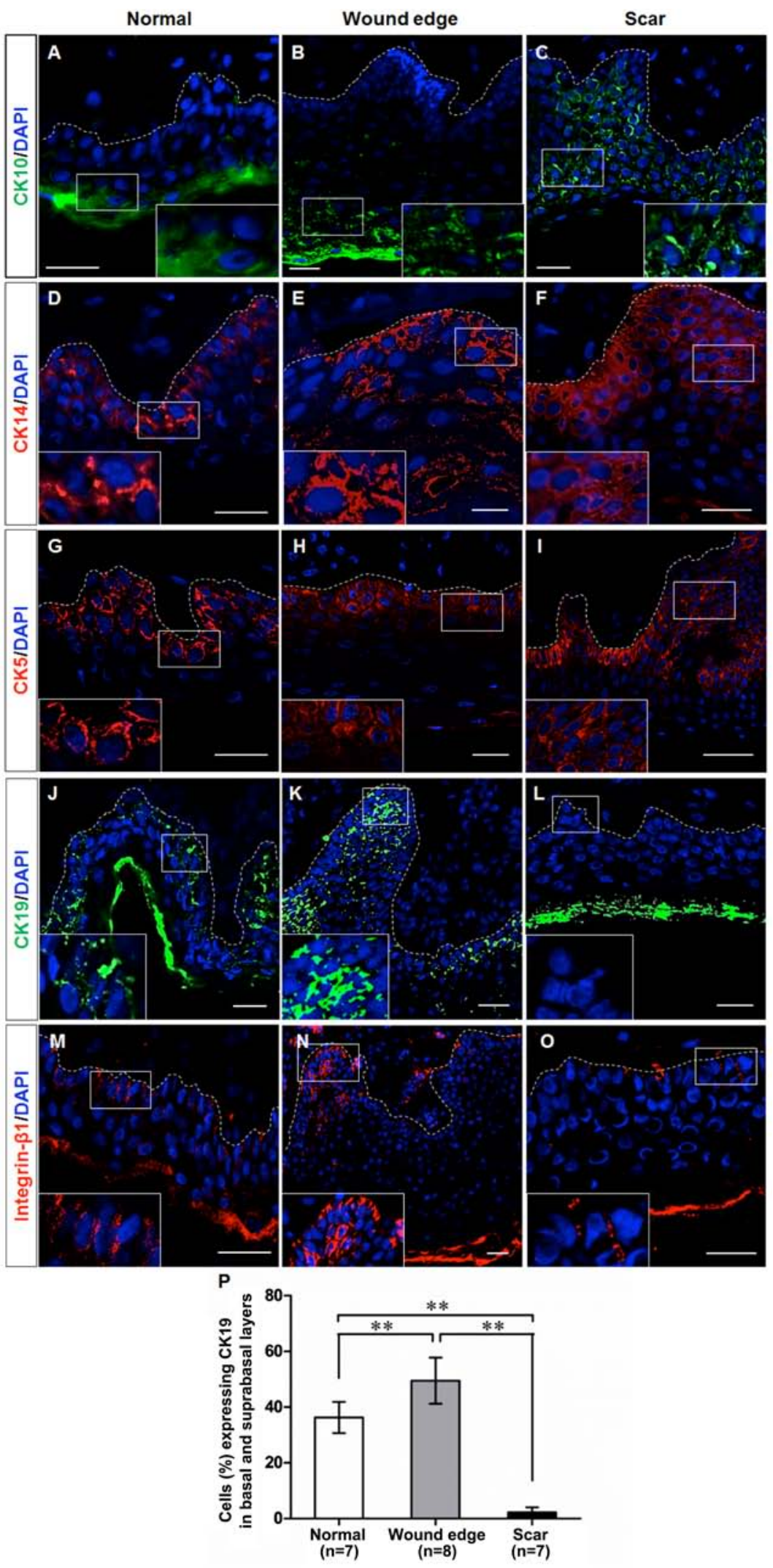

Figure 2. Cell marker expression in epidermal keratinocytes in normal, wound edge and hypertrophic scar tissue. Representative fluorescence images showing the localization of CK10, CK14, CK5, CK19 and integrin- $\beta 1$ in the sections of normal, wound edge and scar tissues. (A-C) CK10, a marker of differentiated keratinocytes, was expressed in the outer layer of the epidermis of (A) normal and (B) wound edge tissues, and in (C) the suprabasal, terminally differentiating cells in the epidermis of hypertrophic scar tissues. (D-F) CK14 was expressed in both basal and suprabasal layers in the stratified epidermis of all three tissues. In particular, extensive distribution of CK14 was detectable in (F) the multilayered epidermis of the scar tissue. (G-I) The distribution patterns of CK5 were similar to those of CK14, which indicated an increased number of proliferating cells in the hyperproliferative epidermis. (J-O) CK19 and integrin- $\beta 1$ were expressed in the basal layer of ( $\mathrm{J}$ and $\mathrm{M})$ normal epidermis, and in the basal and suprabasal layers of (K and $\mathrm{N}$ ) wound edge epidermis, but were not detected in (L and $\mathrm{O}$ ) the epidermis of hypertrophic scar tissues. White dotted lines denote the basement membrane, which separated the epidermis and dermis. Insets show a close-up view of area within the white markings. All scale bars represent $25 \mu \mathrm{m}$. (P) Statistical analysis of CK19-expressing cells in the basal and suprabasal layers in normal, wound edge and hypertrophic scar tissues. Data are the means \pm SD. ${ }^{* * *} \mathrm{P}<0.01$. 

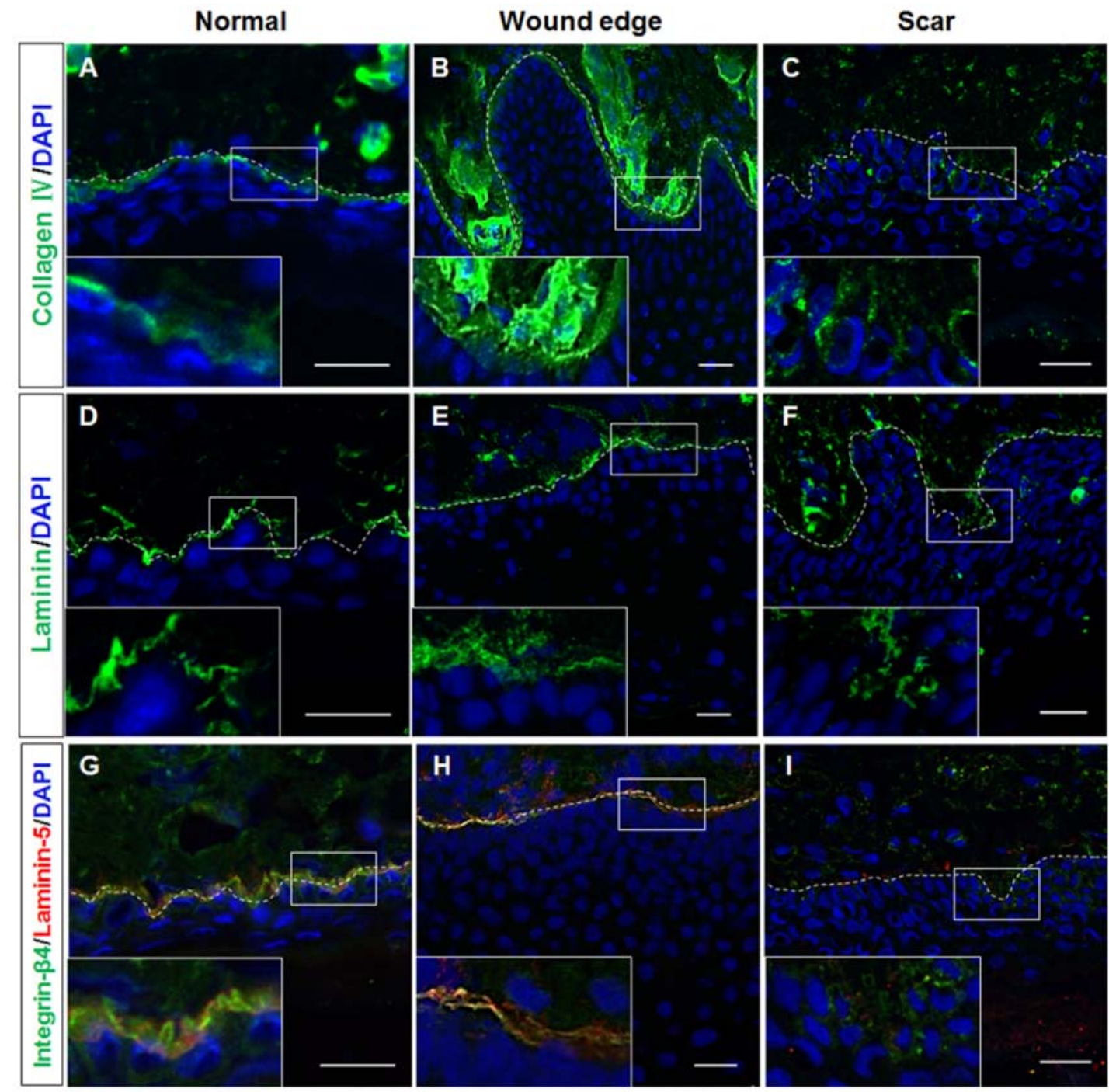

Figure 3. Different distribution patterns of basement membrane (BM) components in normal, wound edge and hypertrophic scar tissue. (A-C) Representative fluorescence images showing the distribution patterns of collagen IV in normal, wound edge and hypertrophic scar tissues. Collagen IV expression was detectable in the BM area in both (A) normal and (B) wound edge tissues. However, collagen IV staining was absent in (C) the epidermis of hypertrophic scar tissues. (D-F) Representative fluorescence images showing the distribution patterns of laminin in normal, wound edge and hypertrophic scar tissues. (G-I) Representative fluorescence images showing the localization of laminin- 5 and integrin- $\beta 4$ in normal, wound edge and hypertrophic scar tissues. Negative staining of both laminin- 5 and integrin- $\beta 4$ was observed in (I) the hypertrophic scar tissues as compared with $(\mathrm{G})$ the normal and (H) wound edge tissues. White dotted lines denote the basement membrane, which separated the epidermis and dermis. Insets show a close-up view of area within the white markings. All scale bars represent $25 \mu$ m.

was observed as compared with that in the normal (Figs. $3 \mathrm{G}$ and $4 \mathrm{~A}-\mathrm{C}$ ) and wound edge tissues (Figs. 3H and 4D-F). Given these findings, it is likely that the normal BM contributed to the recruitment of integrin- $\beta 1$ - and CK19-expressing cells in the basal layer of the normal and wound edge epidermis. Abnormalities in the BM structure, however, induced the basal keratinocytes to differentiate and adopt a proliferative phenotype during scar pathogenesis.

Altered BM structure promotes epidermal keratinocytes to adopt a proliferative phenotype in vitro. The mechanical support provided by the BM is determined primarilybyits type IV collagen scaffold (21), into which the laminin networks and perlecan oligomers are integrated by the cross-linking nidogens to finally assemble the sheet-like BM complex (22). To further clarify the roles of the $\mathrm{BM}$ in the regulation of epidermal keratinocyte behavior, we used the human BM extract-derived ECM to coat the culture dishes in order to control the pattern of cell-matrix adhesion. As shown in Fig. 5, ECM administration resulted in the upregulation of integrin- $\beta 4$ expression in the HEKs (Fig. 5A) and the HaCaT (Fig. 5B) cells, which was present on the plasma membrane and at the sites of cell-matrix attachment. We also performed western blot analysis to examine the protein levels of integrin- $\beta 4$ in the epidermal cell lines with or without ECM coating. Our data demonstratd that higher levels of integrin- $\beta 4$ were detectable in the ECM-treated groups than in the controls, which further confirmed the fluorescent immunostaining findings (Fig. 5C). Moreover, the HEKs and HaCaT cells were switched to medium containing $1.5 \mathrm{mM} \mathrm{Ca}^{2+}$ for the indicated periods of time to generate differentiating epidermal cells as previously described $(23,24)$. Following $\mathrm{Ca}^{2+}$ treatment, the morphological alterations in both the HEKs (Fig. 6A) and 


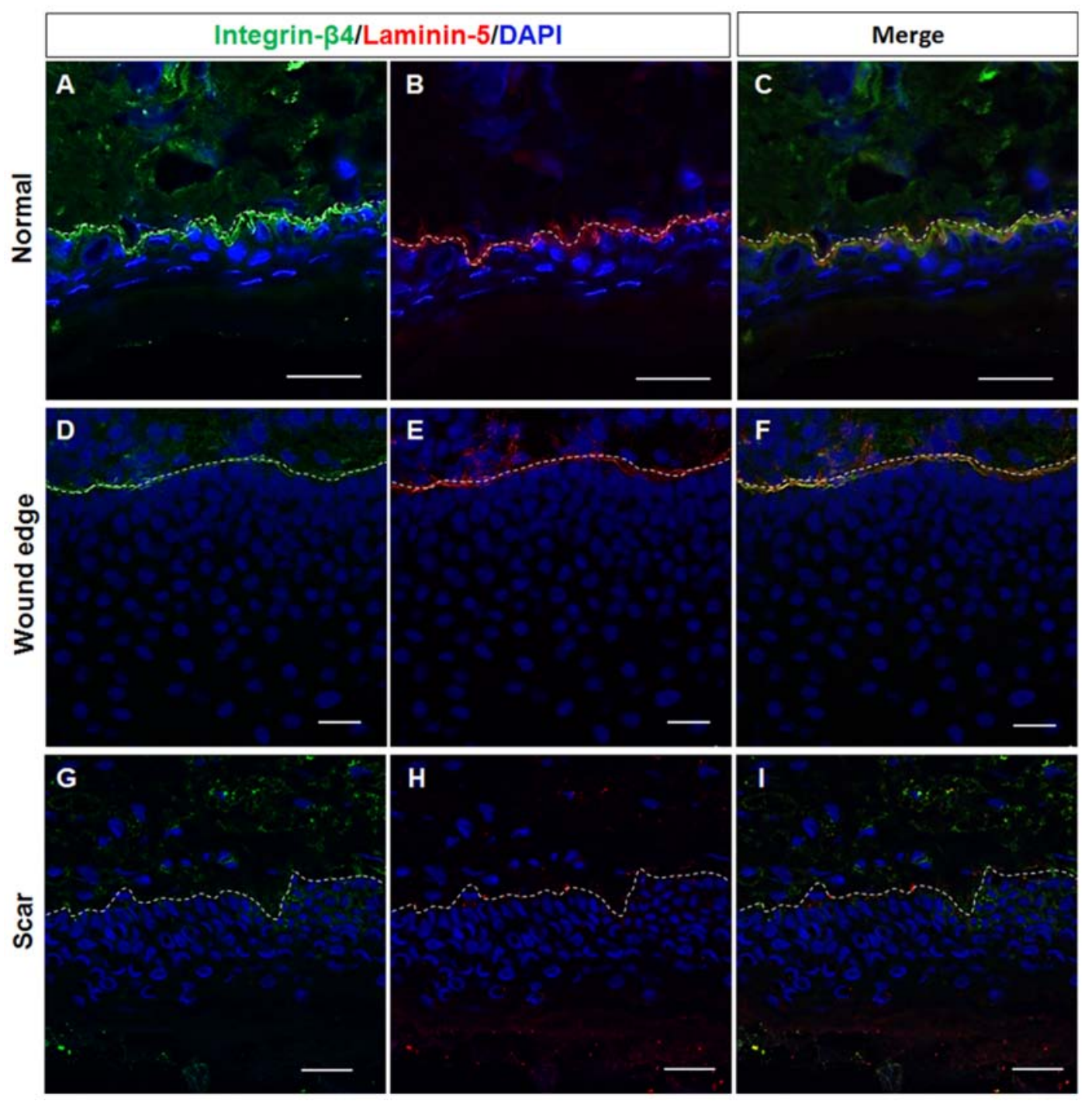

Figure 4. Representative fluorescence images showing the double-labelling of laminin-5 and integrin- $\beta 4$ in normal, wound edge and hypertrophic scar tissue (A-I) The distribution of laminin- 5 and integrin- $\beta 4$ was detected in the basement membrane (BM) area in both (A-C) normal and (D-F) wound edge skin tissues. (G-I) The formation of BM-like structures in the absence of laminin-5 and integrin- $\beta 4$ expression was observed in the epidermis of hypertrophic scar tissues. White dotted lines denote the basement membrane, which separated the epidermis and dermis. All scale bars represent $25 \mu \mathrm{m}$.

HaCaT cells (Fig. 6B) were observed under a light microscope, which included cell enlargement and flattening. $\mathrm{Ca}^{2+}$ induced the expression of CK10 (Figs. 5D, G and J, and 6C and D) and CK14 (Figs. 5E, $\mathrm{H}$ and $\mathrm{J}$, and 6C and D) in a time-dependent manner in epidermal cell lines at both the mRNA (Figs. 5D, E, G and H, and 6C and D) and protein (Fig. 5J) levels, with peak levels of CK10 and CK14 expression being observed $24 \mathrm{~h}$ after initiating $1.5 \mathrm{mM} \mathrm{Ca}^{2+}$ treatment in the HEKs (Figs. 5D, $\mathrm{E}$ and $\mathrm{J}$ and $6 \mathrm{C}$ ) and the HaCaT cells (Figs. 5G, $\mathrm{H}$ and $\mathrm{J}$ and 6D). Following $\mathrm{Ca}^{2+}$ treatment, the expression of CK19 was downregulated in the HEKs, reaching its lowest level at $24 \mathrm{~h}$ after the start of $\mathrm{Ca}^{2+}$ treatment (Figs. 5F and $\mathrm{J}$ and $6 \mathrm{C}$ ). Similar results were observed in the HaCaT cells (Figs. 5I and $\mathbf{J}$ and 6D). ECM treatment, however, reduced the differentiating responses of keratinocytes associated with $\mathrm{Ca}^{2+}$ administration in the HEKs and HaCaT cells, and enhanced the expression of CK19 at $12 \mathrm{~h}$ and $24 \mathrm{~h}$ after $\mathrm{Ca}^{2+}$ treatment (Fig. 5F, I and J). To further determine the potential role of the $\mathrm{BM}$ in the regulation of epidermal keratinocyte behavior, type IV collagen was used to form a BM-like structure in vitro. Collagen IV treatment contributed to CK19 expression and reduced the expression of CK10 and CK14 at the mRNA and protein levels in both the HEKs (Fig. 7A-C and G) and the HaCaT cells (Fig. 7D-F and $\mathrm{G})$. These results further validated our in vivo findings that the BM appears to be a positive regulator of the recruitment of precursor-like cells in the basal layer of the epidermis. Alterations in the BM structure promoted the development of a proliferative phenotype in basal keratinocytes as indicated by the enhanced expression of the markers associated with cell proliferation, e.g., CK14 and CK5.

\section{Discussion}

In the present study, we first investigated the morphological changes which occur during epidermal regeneration as part of the wound healing process. Our data demonstrated that the 

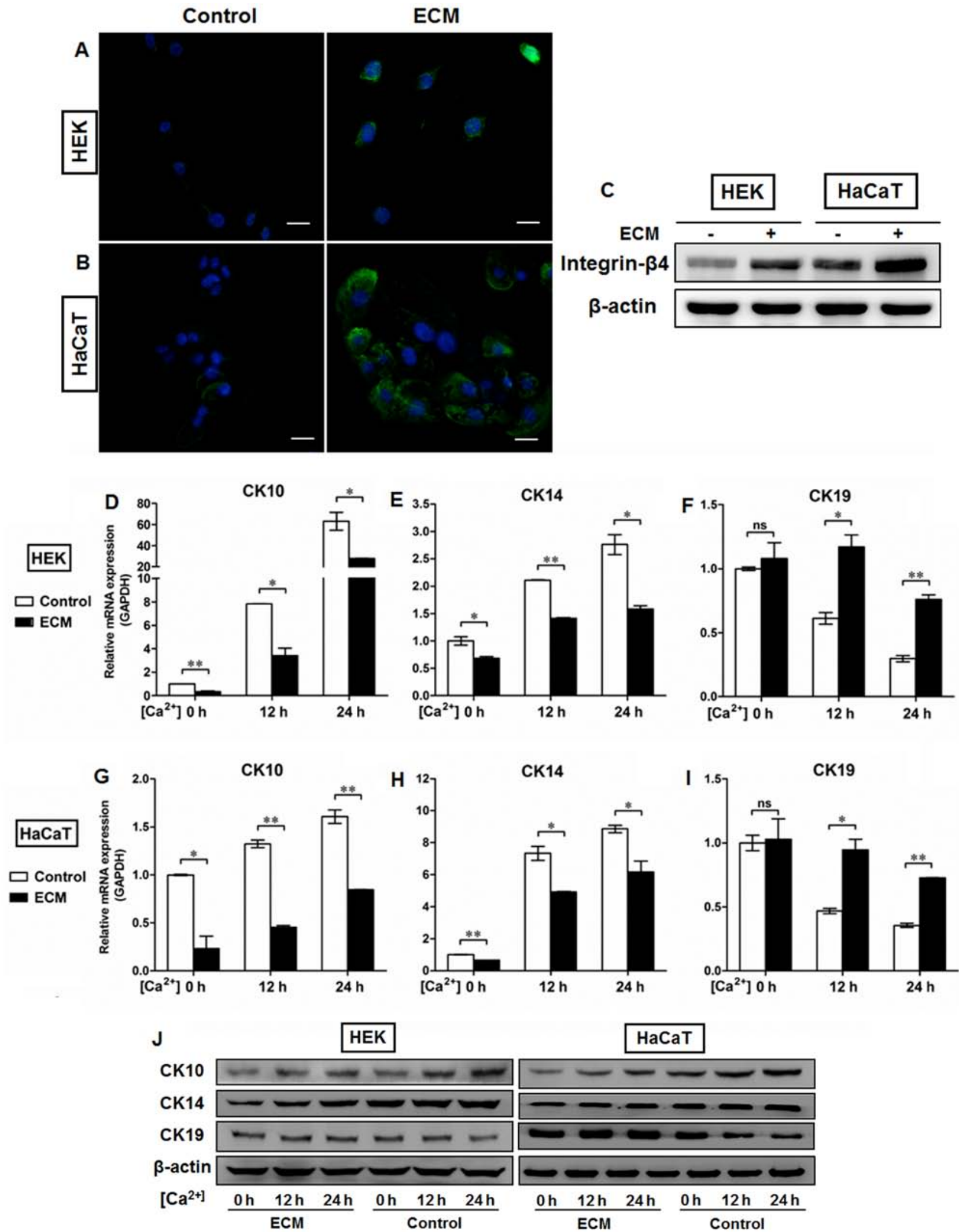

Figure 5. Extracellular matrix (ECM) coating regulates the expression of cytokeratin (CK)10, CK14 and CK19 in epidermal keratinocytes in vitro. Human basement membrane (BM) extract-derived ECM was used to minic the BM structure in vitro. (A and B) Representative fluorescence images showing the localization of integrin- $\beta 4$ (green) in (A) human epidermal keratinocytes (HEKs) and (B) human immortalized keratinocyte (HaCaT) cells with or without ECM coating. Nuclei were stained with DAPI (blue). All scale bars represent $25 \mu \mathrm{m}$. (C) Western blot analysis of integrin- $\beta 4$ expression in HEKs and HaCaT cells with or without ECM coating. (D-I) After primary cultured HEKs and $\mathrm{HaCaT}$ cells were switched to the medium containing $1.5 \mathrm{mM} \mathrm{Ca}^{2+}$ for the indicated time periods, the relative levels of CK10, CK14, and CK19 expression were assessed by real-time qPCR. Ca ${ }^{2+}$ induced the expression of (D and G) CK10 and (E and H ) CK14 in a timedependent manner in the epidermal cell lines, with a peak expression level $24 \mathrm{~h}$ after $1.5 \mathrm{mM} \mathrm{Ca}^{2+}$ treatment in (D and E) $\mathrm{HEKs}$ and (G and $\left.\mathrm{H}\right) \mathrm{HaCaT}$ cells. ECM treatment, however, reduced the differentiating responses of keratinocytes associated with $\mathrm{Ca}^{2+}$ administration in (F) HEKs and (I) HaCaT cells, and enhanced expression of $\mathrm{CK} 19$ at $12 \mathrm{~h}$ and $24 \mathrm{~h}$ after $\mathrm{Ca}^{2+}$ addition. Data are the means $\pm \mathrm{SD} .{ }^{*} \mathrm{P}<0.05,{ }^{* *} \mathrm{P}<0.01, \mathrm{~ns}=$ not significant. (J) Western blot analysis of CK10, CK14, and CK19 expression in HEKs and HaCaT cells with or without ECM coating. 
$\left[\mathrm{Ca}^{2+}\right]$
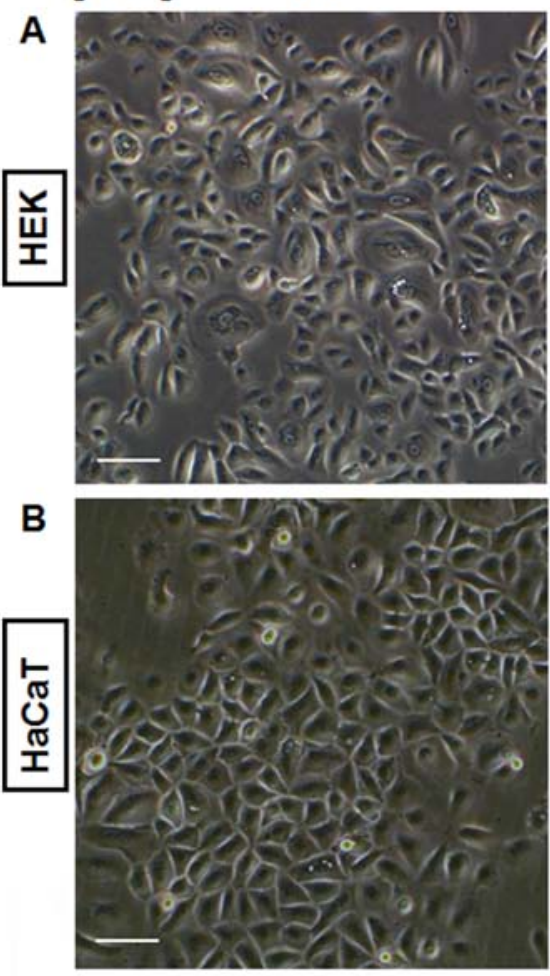

C

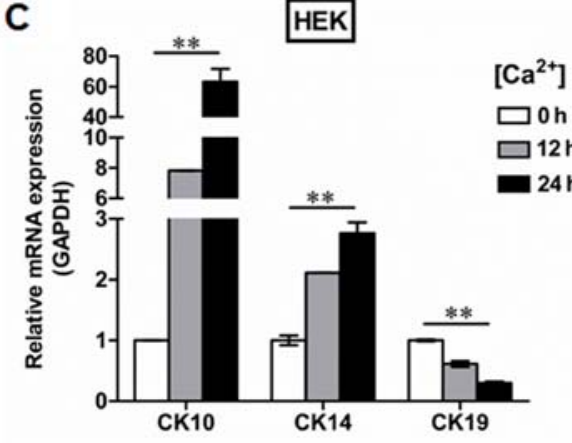

$12 \mathrm{~h}$
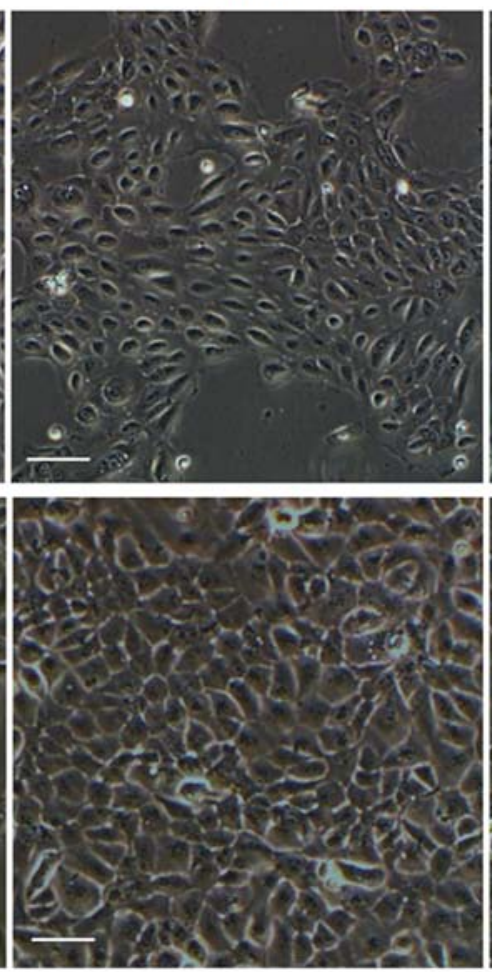

$24 \mathrm{~h}$
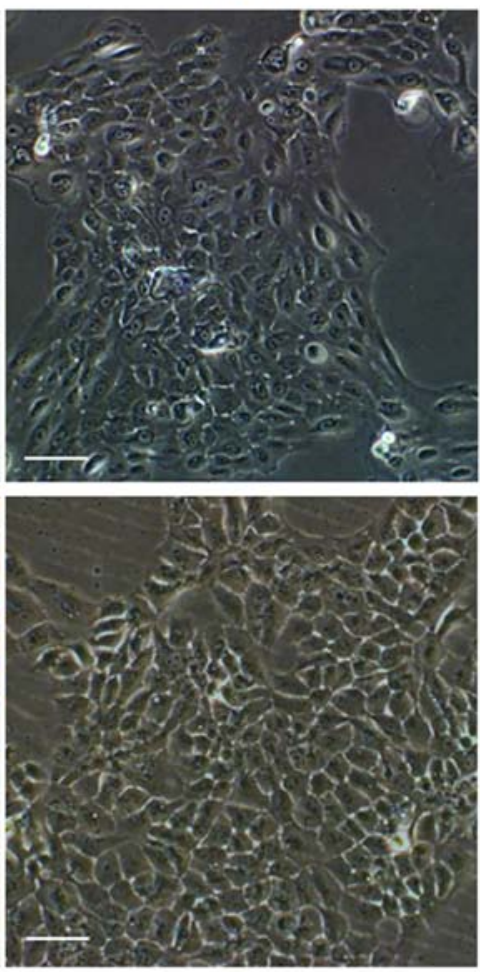

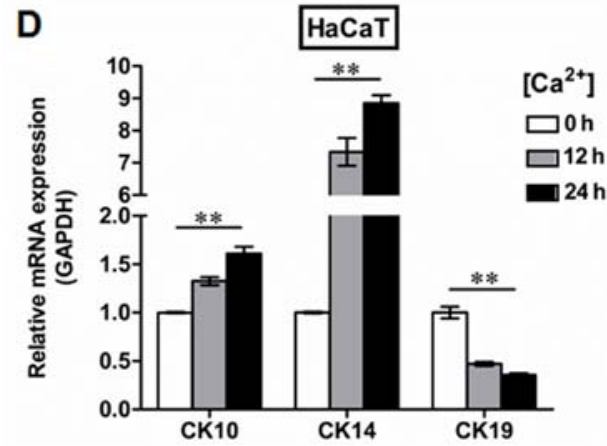

Figure 6. Generation of a differentiation model of epidermal cells in vitro. Human epidermal keratinocytes (HEKs) and human immortalized keratinocyte (HaCaT) cells were switched to the medium containing $1.5 \mathrm{mM} \mathrm{Ca}^{2+}$ for the indicated time periods to generate differentiating epidermal cells. Morphological changes in (A) HEKs and (B) HaCaT cells after $\mathrm{Ca}^{2+}$ treatment for 0,12 , and $24 \mathrm{~h}$. After switching the cells to the medium containing $1.5 \mathrm{mM} \mathrm{Ca}{ }^{2+}$, both (A) $\mathrm{HEKs}$ and (B) HaCaT cells became enlarged and flattened. All scale bars represent $100 \mu \mathrm{m}$. (C and D) RT-qPCR was performed to detect the expression of CK10, CK14 and CK19 in (C) HEK and (D) $\mathrm{HaCaT}$ cells after $\mathrm{Ca}^{2+}$ treatment for the indicated time periods. Our data showed that $\mathrm{Ca}^{2+}$ treatment upregulated CK10 and CK14 mRNA levels in a time-dependent manner in epidermal keratinocytes, whereas the mRNA levels of CK19 decreased significantly in response to $\mathrm{Ca}^{2+}$ stimulation. ${ }^{* *} \mathrm{P}<0.01$.

histological structure of the hypertrophic scar tissues differed from that of normal skin tissue, with a significant increase in epidermal thickness between the basal layer and stratum corneum being observed. Notably, staining of the BM appeared to be absent in the scar tissue. Moreover, immunofluorescence staining for CK10, CK14, CK5, CK19 and integrin- $\beta 1$ indicated that the differential expression of basal keratinocyte markers differed among the samples of normal, wound edge and hypertrophic scar tissues, and furthermore the epidermis of the hypertrophic scar tissue exhibited a proliferative phenotype by switching the expression of integrin- $\beta 1$ and CK19 to CK14 and CK5, markers of cell proliferation, in the multilayered epidermis. Given these findings, we thus hypothesized that the BM plays a role in regulating the cell fate decision of epidermal keratinocytes during wound healing. By using a panel of antibodies associated with BM components, we validated our hypothesis that the structure of the BM was altered in the hypertrophic scar tissues. Our results indicated that the BM contributed to the recruitment of CK19-expressing cells in the basal layer of normal and wound edge epidermis, whereas abnormalities in the structure of the BM induced the basal keratinocytes to differentiate and adopt a proliferative phenotype during scar pathogenesis. By using ECM and collagen IV to mimic the BM structure in vitro, we further confirmed our in vivo observations and showed that the $\mathrm{BM}$ reduced the differentiating responses of keratinocytes induced by $\mathrm{Ca}^{2+}$ administration in the epidermal cell lines and enhanced the expression of CK19, a putative marker of epidermal progenitor cells.

The BM plays a fundamental role in the differentiation, proliferation, survival and migration of cells during embryonic 


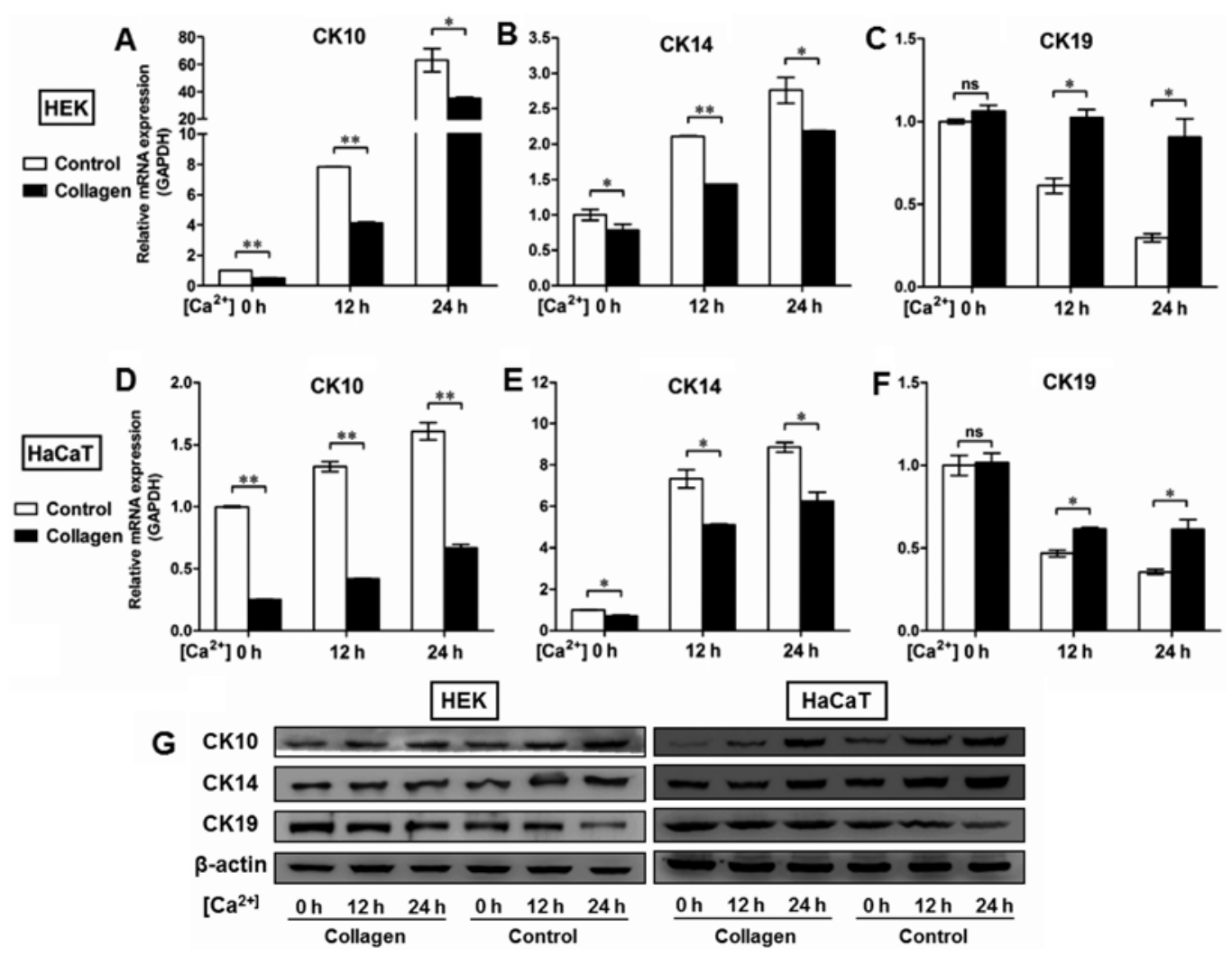

Figure 7. Type IV collagen coating regulates the expression of CK10, CK14 and CK19 in epidermal keratinocytes in vitro. (A-F) To further determine the potential role of basement membrane (BM) in the regulation of epidermal keratinocyte behavior, type IV collagen was used to form a BM-like structure in vitro. After human epidermal keratinocytes (HEKs) and human immortalized keratinocyte (HaCaT) cells were switched to the medium containing $1.5 \mathrm{mM} \mathrm{Ca}^{2+}$, the relative levels of CK10, CK14, and CK19 expression were assessed by RT-qPCR. Although $\mathrm{Ca}^{2+}$ contributed to the expression of (A and D) CK10 and (B and E) CK14 in a time-dependent manner in epidermal cell lines, type IV collagen treatment reduced the mRNA expression of CK10 and CK14 in both (A and B) HEKs and (D and E) HaCaT cells. Type IV collagen treatment also increased the mRNA levels of CK19 in (C) HEKs and (F) HaCaT cells in vitro. Data are the means \pm SD. ${ }^{*} \mathrm{P}<0.05,{ }^{* *} \mathrm{P}<0.01$, ns, not significant. (G) Western blot analysis of CK10, CK14, and CK19 expression in HEKs and HaCaT cells with or without collagen IV treatment.

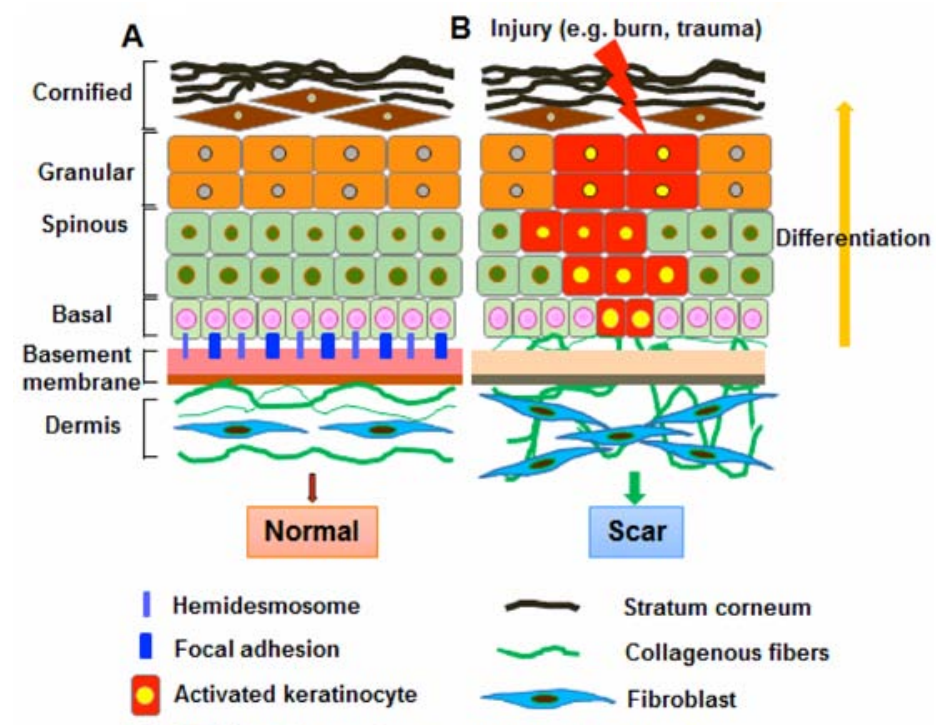

Figure 8. Schematic representation of the regulatory roles of basement membrane (BM) in maintaining basal keratinocyte function in skin wound healing. (A) In normal skin, BM separates the epidermis and dermis and functions as a critical regulator in the cellular and molecular communication between dermal fibroblasts and epidermal keratinocytes. Stem cells in the basal layer are capable of self-renewal throughout life and the production of daughter cells [transit amplifying cells (TAs)] that undergo differentiation. Basal keratinocytes attach to the BM by hemidesmosomes and adhesion molecules such integrins. (B) Once the skin is injured, basal keratinocytes become activated and migrate into the wound area. The BM then not only serves as a structural scaffold, but contributes to the recruitment of epidermal progenitor cells in the basal layer of skin epidermis. The recruitment of epidermal progenitor cells in the basal layer helps maintain the balance between basal keratinocyte renewal and differentiation during wound repair and regeneration. Alterations in BM structure, however, reduces the attachment of basal progenitor cells to their surrounding microenvironment, and induces them to differentiate and adopt a proliferating phenotype, which may one of the initiators of scar development. 
development. In the skin, the BM physically separates the epidermis from the underlying dermis. The basal keratinocytes in the epidermis attach to the underlying BM through integrins, which bind to some BM components, such as collagen, laminin and fibronectin (25). Thus, the BM not only serves as a selective barrier and structural scaffold, but also provides a communication interface between dermal fibroblasts and epidermal keratinocytes $(2,26)$. The BM also binds to a number of cytokines and growth factors, serving as a reservoir for their controlled release, which regulates keratinocyte-fibroblast interactions to promote the development of skin, as well as wound healing $(27,28)$. Previous studies on scar formation have mainly focused on fibroblasts and little is known about the role of the overlying epidermal keratinocytes $(29,30)$. There is growing evidence that keratinocytes may play an important role in the development of pathological fibrosis through paracrine regulation of fibroblast function $(6,9,10)$. It has also been shown that keratinocytes derived from scar tissues differed from normal keratinocytes by exhibiting differential gene expression profiles (12). The mechanisms responsible for the fundamental abnormalities in keratinocytes during keloid and hypertrophic scarring remain elusive. Our data indicated a potential link between BM remodeling and the maintenance of keratinocyte function during wound repair and regeneration. It is well known that adhesion molecules, such integrins and E- and $\mathrm{N}$-cadherin, anchor stem cells to the ECM (22). In light of our observations, it appears that the BM may represent part of the niche components for the recruitment of epidermal progenitor cells in the basal layer of the skin epidermis. Our results indicate that the abnormal functioning of the BM reduces the attachment of basal progenitor cells to their surrounding microenvironment, and induces them to adopt a proliferative phenotype, which may account for the pathogenesis of scarring (Fig. 8).

\section{Acknowledgements}

This study was supported in part by grants from the Novel Program of Beijing (nos. 2008B53 and 2009A38) and the National Natural Science Foundation of China (nos. 30901564, $81101883,81372067,81121004$ and 81230041) and the National Basic Science and Development Program (973 Program, 2012CB518105).

\section{References}

1. Fuchs E and Raghavan S: Getting under the skin of epidermal morphogenesis. Nat Rev Genet 3: 199-209, 2002

2. Breitkreutz D, Mirancea N and Nischt R: Basement membranes in skin: unique matrix structures with diverse functions? Histochem Cell Biol 132: 1-10, 2009.

3. Blanpain C and Fuchs E: Epidermal stem cells of the skin. Annu Rev Cell Dev Biol 22: 339-373, 2006.

4. Snippert HJ, Haegebarth A, Kasper M, Jaks V, van Es JH, Barker N, van de Wetering M, van den Born M, Begthel H, Vries RG, et al: Lgr6 marks stem cells in the hair follicle that generate all cell lineages of the skin. Science 327: 1385-1389, 2010.

5. Broughton G II, Janis JE and Attinger CE: The basic science of wound healing. Plast Reconstr Surg 117 (Suppl 7): 12S-34S, 2006.

6. Bellemare J, Roberge CJ, Bergeron D, Lopez-Vallé CA, Roy M and Moulin VJ: Epidermis promotes dermal fibrosis: role in the pathogenesis of hypertrophic scars. J Pathol 206: 1-8, 2005.

7. Andriessen MP, Niessen FB, Van de Kerkhof PC and Schalkwijk J: Hypertrophic scarring is associated with epidermal abnormalities: an immunohistochemical study. J Pathol 186: 192-200, 1998.
8. Teepe RG, Kreis RW, Koebrugge EJ, Kempenaar JA, Vloemans AF, Hermans RP, Boxma H, Dokter J, Hermans J, Ponec M, et al: The use of cultured autologous epidermis in the treatment of extensive burn wounds. J Trauma 30: 269-275, 1990.

9. Funayama E, Chodon T, Oyama A and Sugihara T: Keratinocytes promote proliferation and inhibit apoptosis of the underlying fibroblasts: an important role in the pathogenesis of keloid. J Invest Dermatol 121: 1326-1331, 2003.

10. Lim IJ, Phan TT, Bay BH, Qi R, Huynh H, Tan WT, Lee ST and Longaker MT: Fibroblasts cocultured with keloid keratinocytes: normal fibroblasts secrete collagen in a keloidlike manner. Am J Physiol Cell Physiol 283: C212-C222, 2002.

11. Hahn JM, Glaser K, McFarland KL, Aronow BJ, Boyce ST and Supp DM: Keloid-derived keratinocytes exhibit an abnormal gene expression profile consistent with a distinct causal role in keloid pathology. Wound Repair Regen 21: 530-544, 2013.

12. Machesney M, Tidman N, Waseem A, Kirby L and Leigh I: Activated keratinocytes in the epidermis of hypertrophic scars. Am J Pathol 152: 1133-1141, 1998.

13. Orazizadeh M, Hashemitabar M, Bahramzadeh S, Dehbashi FN and Saremy S: Comparison of the enzymatic and explant methods for the culture of keratinocytes isolated from human foreskin. Biomed Rep 3: 304-308, 2015.

14. Su L, Morgan PR and Lane EB: Keratin 14 and 19 expression in normal, dysplastic and malignant oral epithelia. A study using in situ hybridization and immunohistochemistry. J Oral Pathol Med 25: 293-301, 1996.

15. Michel M, Török N, Godbout MJ, Lussier M, Gaudreau P, Royal A and Germain L: Keratin 19 as a biochemical marker of skin stem cells in vivo and in vitro: keratin 19 expressing cells are differentially localized in function of anatomic sites, and their number varies with donor age and culture stage. J Cell Sci 109: 1017-1028, 1996.

16. Khanom R, Sakamoto K, Pal SK, Shimada Y, Morita K, Omura K, Miki Y and Yamaguchi A: Expression of basal cell keratin 15 and keratin 19 in oral squamous neoplasms represents diverse pathophysiologies. Histol Histopathol 27: 949-959, 2012.

17. Fuchs E: Skin stem cells: rising to the surface. J Cell Biol 180: 273-284, 2008

18. Yeh YC, Lin HH and Tang MJ: A tale of two collagen receptors, integrin $\beta 1$ and discoidin domain receptor 1, in epithelial cell differentiation. Am J Physiol Cell Physiol 303: C1207-C1217, 2012.

19. Borowska K, Jedrych B, Czerny K and Zabielski S: The role of integrins in the physiologic and pathogenic processes. Pol Merkur Lekarski 21: 362-366, 2006 (Article in Polish).

20. Lane SW, Williams DA and Watt FM: Modulating the stem cell niche for tissue regeneration. Nat Biotechnol 32: 795-803, 2014.

21. Breitkreutz D, Koxholt I, Thiemann K and Nischt R: Skin basement membrane: the foundation of epidermal integrity - BM functions and diverse roles of bridging molecules nidogen and perlecan. Biomed Res Int 2013: 179784, 2013.

22. Timpl R and Brown JC: Supramolecular assembly of basement membranes. BioEssays 18: 123-132, 1996.

23. Hennings H, Michael D, Cheng C, Steinert P, Holbrook K and Yuspa SH: Calcium regulation of growth and differentiation of mouse epidermal cells in culture. Cell 19: 245-254, 1980.

24. Hennings $\mathrm{H}$ and Holbrook KA: Calcium regulation of cell-cell contact and differentiation of epidermal cells in culture. An ultrastructural study. Exp Cell Res 143: 127-142, 1983.

25. Burgeson RE and Christiano AM: The dermal-epidermal junction. Curr Opin Cell Biol 9: 651-658, 1997.

26. LeBleu VS, Macdonald B and Kalluri R: Structure and function of basement membranes. Exp Biol Med (Maywood) 232: 1121-1129, 2007.

27. Iozzo RV: Basement membrane proteoglycans: from cellar to ceiling. Nat Rev Mol Cell Biol 6: 646-656, 2005.

28. Werner S, Krieg T and Smola H: Keratinocyte-fibroblast interactions in wound healing. J Invest Dermatol 127: 998-1008, 2007.

29. Moulin V,Castilloux G, Auger FA, GarrelD, O'Connor-McCourt M and Germain L: Modulated response to cytokines of human wound healing myofibroblasts compared to dermal fibroblasts. Exp Cell Res 238: 283 -293, 1998.

30. Gabbiani G, Ryan GB and Majne G: Presence of modified fibroblasts in granulation tissue and their possible role in wound contraction. Experientia 27: 549 -550, 1971. 